\title{
Fatores maternos para o nascimento de recém-nascidos com baixo peso e prematuros: estudo caso-controle
}

\author{
Maternal factors for the birth of newborns with low birth weight and premature: \\ case-control study
}

\author{
Silas Santos Carvalho, , Julita Maria Freitas Coelho ${ }^{\mathrm{b}}$, Denise Ângela Bacelarc, Elayne Mariolac \\ a Enfermeiro. Pós-graduação em Obstetrícia e Saúde da Mulher. Mestrando em Saúde Coletiva, Universidade Estadual de Feira de Santana (BA). \\ b Dentista. Doutora em Saúde Pública. Universidade Estadual de Feira de Santana (BA). \\ c Acadêmica do Curso de Enfermagem, Faculdade Anísio Teixeira de Feira de Santana (BA).
}

RESUMO Objetivo: Identificar os fatores maternos mais associados ao nascimento de recém-nascidos com baixo peso e prematuros.

Materiais e Métodos: Estudo caso-controle com 111 puérperas que buscaram atendimento em um hospital público de Feira de Santana (BA). O grupo caso foi constituído por mães de neonatos com baixo peso $(<2500 \mathrm{~g})$ e $<37$ semanas, e o grupo controle, por mães de neonatos com peso normal ( $\geq 2500 \mathrm{~g}$ ) e a termo. O peso ao nascer e idade gestacional foram obtidos através do registro hospitalar. Os principais fatores de risco para o BPN e prematuridade foram avaliados mediante análise bivariada e obtenção da medida de associação odds ratio (OR).

Resultados: $18,9 \%$ das puérperas tiveram filhos prematuros e, em 38,1\% dos casos, o parto ocorreu antes de 34 semanas. A maioria dessas mulheres tinha idade entre 14 e 25 anos $(66,7 \%)$, cor negra ou parda $(85,7 \%)$, com companheiro $(81,0 \%)$, tiveram o parto normal $(57,0 \%)$ e visitaram o dentista durante a gestação $(95,2 \%)$. Houve associação estatisticamente significante entre baixo peso ao nascer/parto prematuro e o consumo de bebida alcóolica (OR: 7,9; $p=0,001)$, número de consultas pré-natais menor que 6 (OR: 2,94; $p=0,02)$ e uso de medicação abortiva (OR: 5,$05 ; p=0,04)$.

Conclusão: Os achados demonstraram que as mulheres que consumiam bebida alcoólica, que realizaram menos de seis consultas pré-natais e usavam medicação abortiva, tiveram maior chance do bebê nascer com baixo peso e prematuro. Assim, esses fatores de risco merecem atenção especial nas políticas públicas para o enfrentamento desse agravo.

Palavras-chave: baixo peso ao nascer; prematuridade; mortalidade infantil; epidemiologia.

Objective: To identify maternal factors most associated with the birth of low weight and premature infants.

Materials and Methods: Case-control study with 111 mothers who sought care at a public hospital in Feira de Santana (BA). The case group consisted of mothers of newborns with low birth weight $(<2500 \mathrm{~g})$ and $<37$ weeks; and the control group of mothers of newborns with normal weight $(\geq 2500 \mathrm{~g})$ and term. Birth weight and gestational age were obtained from hospital records. The main risk factors for low birth weight and prematurity were assessed by bivariate analysis and by calculating the odds ratio (OR).

Results: $18.9 \%$ of mothers had preterm deliveries and, in $38.1 \%$ of cases, delivered before 34 weeks. Most of these women were aged between 14 and 25 years (66.7\%), black or mulatto (85.7\%), had a partner (81.0\%), vaginal delivery (57.0\%) and visited dentist during pregnancy (95.2\%). There was a statistically significant association between low birth weight/premature delivery and consumption of alcoholic beverages (OR: 7.9; $p=0.001)$, number of antenatal visits less than $6(\mathrm{OR}: 2.94 ; p=0.02)$ and the use of abortive medication (OR: $5.05 ; p=0.04$ ).

Conclusion: The findings showed that women who consumed alcohol, had fewer than six prenatal consultations and used abortive medication, had a greater chance of delivering a baby with low birth weight and premature. Thus, these risk factors deserve special attention in public policies to face this disease.

Keywords: low birth weight; prematurity; child mortality; epidemiology. 


\section{INTRODUÇÃO}

A Organização Mundial da Saúde (OMS) ${ }^{1}$ define o baixo peso ao nascer (BPN) como todo nascido vivo com menos de $2500 \mathrm{~g}$, sendo um importante determinante da mortalidade infantil. Essa condição leva a um maior risco de infecções, propensão ao retardo de crescimento e déficit neuropsicológico podendo repercutir nas condições de vida do adulto $^{1-3}$. Em 2007, a mortalidade neonatal (óbito no período de 0 a 27 dias de vida) representou $70 \%$ da mortalidade infantil no país e o componente neonatal precoce (óbito no período de 0 a 6 dias de vida) foi responsável por $50 \%$ dessas mortes $^{4-6}$. Não obstante os inúmeros estudos publicados sobre a temática, os determinantes para o BPN não estão inteiramente instituídos, faltam achados mais consistentes, principalmente em países em desenvolvimento ${ }^{1,3}$.

Já a prematuridade refere-se à condição, segundo a Organização Mundial da Saúde (OMS), de bebês que nascem entre 20 e 37 semanas de gestação. De acordo a UNICEF , em todo o mundo nascem anualmente 20 milhões de crianças prematuras ou com baixo peso. No Brasil, um terço destes recém-nascidos (RN) morre antes do primeiro ano de vida, devido a infecções e problemas respiratórios, os quais são reconhecidos como primeira causa de mortalidade infantil no país ${ }^{8}$. Os fatores que influenciam tais desfechos gestacionais negativos interrelacionam de forma complexa e se originam de condições biológicas, sociais e ambientais, que a mulher está exposta durante a gestação $0^{7,9-11}$. É importante destacar que cerca de $40 \%$ e $50 \%$ dos nascimentos prematuros ainda têm causas desconhecidas ou idiopáticas ${ }^{12}$.

Segundo Ebersole et al. ${ }^{2}$ e Cruz et al. ${ }^{3}$, a ocorrência de baixo peso ao nascer e prematuridade está associada a condições socioeconômicas desfavoráveis, nutrição inadequada, primiparidade, hábito de fumar e alcoolismo durante a gravidez, extremos de idade, baixo índice de massa corpórea (IMC) pré-gestacional e complicações no período gestacional, dentre outros fatores.

Diante do exposto faz-se necessário uma melhor compreensão acerca dos mecanismos pelos quais os fatores maternos se relacionam na gênese desse cenário, bem como suas possíveis complicações. Assim, os achados desse estudo poderão favorecer na identificação de novas abordagens do profissional em relação às gestantes. Essas últimas poderão também ser diretamente beneficiadas, visto que um melhor autoconhecimento do seu estado de saúde pode ser fundamental para adoção de condutas mais adequadas e prevenção de agravos no período gestacional.

Portanto, diante da relevância do BPN e da prematuridade para a saúde pública e por se tratar de um tema ainda controverso, a proposta deste estudo foi identificar os fatores maternos mais associados ao nascimento de recém-nascidos com baixo peso e prematuros de uma instituição hospitalar de um município do interior da Bahia.

\section{MATERIAIS E MÉTODOS}

Foi realizado um estudo do tipo caso-controle, no qual foram avaliados dados referentes às mulheres atendidas no Hospital Inácia Pinto dos Santos de Feira de Santana, município do interior da Bahia, para realização de parto no período compreendido entre 29 de junho a 31 de julho de 2015. Ressalta-se que tal instituição atendia prioritariamente pelo Sistema Único de Saúde. Foram elegíveis para a pesquisa todas as mulheres que compareceram ao serviço para realizar o parto, no período da coleta, e que tinham o peso ao nascer do bebê e a idade gestacional disponíveis nos registros de nascimento do hospital e prontuários. O grupo caso foi constituído de 21 mães de crianças nascidas com $<2500$ g e com idade gestacional $<37$ semanas e o grupo controle, por sua vez, foi composto por 90 mães de recémnascidos com peso igual ou superior a $2500 \mathrm{~g}$ e com idade gestacional $\geq 37$ semanas.

Essa amostra de conveniência foi composta unicamente por aquelas que aceitaram participar do estudo e assinaram o Termo de Consentimento Livre e Esclarecido (TCLE). Essa pesquisa foi aprovada pelo Comitê de Ética em Pesquisa (CEP) da Faculdade Anísio Teixeira (FAT) de Feira de SantanaBA, protocolada sob o CAAE no 40126414.9.0000.5631, parecer 1.078.653.

Todas as participantes responderam a um questionário que incluiu dados sociodemográficos, cuidados com a saúde geral e bucal, história gestacional, qualidade do pré-natal e hábitos de vida. Para obtenção de informações complementares, os dados foram coletados em prontuários e cartão de prénatal de todas as participantes. A história médica das gestantes foi revista, não sendo incluídas no estudo aquelas que apresentaram alteração sistêmica que necessitasse de profilaxia antibiótica para os procedimentos odontológicos e internamento hospitalar pós-parto superior a sete dias.

Os dados foram tabulados no programa SPSS 17.0 (Statistical Package for Social Science) e analisados no programa STATA versão 10.0 e versão 11.0. Para a avaliação das associações foi realizada análise estratificada para obtenção das medidas brutas de Odds Ratio (OR). Para avaliar o grau de homogeneidade ou comparabilidade entre os grupos, foi empregado o teste $\chi^{2}$ (Qui-Quadrado) de Pearson e o teste Exato de Fischer quando a frequência esperada foi menor do que 5, com nível de significância de $5 \%$ e intervalo de confiança (IC) de $95 \%(p<0,05)$. 


\section{RESULTADOS}

No total foram investigadas 111 puérperas, seus prontuários e de seus respectivos recém-nascidos analisados. Dentre estas, $21(18,9 \%)$ mulheres compuseram o grupo caso (mães de RN com peso inferior a $2500 \mathrm{~g}$ e com idade gestacional abaixo de 37 semanas), e $90(81,1 \%)$ mulheres o grupo (mães de RN com peso igual ou superior a $2500 \mathrm{~g}$ e com mais de 37 semanas de gestação).

A maioria das mães de bebês com baixo peso e prematuros tinha idade entre 14 e 25 anos $(66,7 \%)$, cor negra ou parda $(85,7 \%)$, com companheiro $(81,0 \%)$ e realizou parto normal $(57,0 \%)$. Quando comparadas com as mães que tiveram filhos com peso normal e que nasceram com mais de 37 semanas, não foram encontradas diferenças estatisticamente significantes para as variáveis estudadas, exceto para o consumo de bebida alcoólica, indicando que existe homogeneidade entre os grupos estudados. Nas puérperas que consumiram álcool durante a gestação, a chance de ter o filho com BPN foi 7,9 vezes maior do que naquelas que não referiram tal consumo (Tabela 1).

Tabela 1. Características sociodemográficas, obstétrica e de estilo de vida dos grupos caso e controle (n=111).

\begin{tabular}{|c|c|c|c|c|}
\hline \multirow{2}{*}{ Características } & Casos $(n=21)$ & Controles $(n=90)$ & \multirow{2}{*}{ OR } & \multirow{2}{*}{$\mathbf{p}^{*}$} \\
\hline & n (\%) & n (\%) & & \\
\hline \multicolumn{5}{|l|}{ Idade (em anos) } \\
\hline $14-25$ & $14(66,7)$ & $71(78,9)$ & 1,00 & \\
\hline $26-47$ & $07(33,3)$ & $19(21,1)$ & 1,86 & $0,36^{* *}$ \\
\hline \multicolumn{5}{|l|}{ Raça/Cor Materna } \\
\hline Branca/amarela & $03(14,3)$ & $05(5,6)$ & 1,00 & \\
\hline Negra/parda & $18(85,7)$ & $85(94,4)$ & 0,35 & $0,34^{* *}$ \\
\hline \multicolumn{5}{|l|}{ Nível de escolaridade (anos estudo) } \\
\hline $0-3$ & $01(4,8)$ & $06(6,7)$ & 1,42 & $1,00 * *$ \\
\hline$\geq 4$ & $20(95,2)$ & $84(93,3)$ & 1,00 & \\
\hline \multicolumn{5}{|l|}{ Renda (salário mínimo) } \\
\hline$<1$ & $05(23,8)$ & $23(25,5)$ & 0,91 & $0,86^{\ddagger}$ \\
\hline$\geq 1$ & $16(76,2)$ & $67(74,5)$ & 1,00 & \\
\hline \multicolumn{5}{|l|}{ Ocupação Materna durante a Gestação } \\
\hline Empregada & $04(19,0)$ & $26(28,9)$ & 1,00 & \\
\hline Desempregada & $17(81,0)$ & $64(71,1)$ & 0,57 & $0,36^{\ddagger}$ \\
\hline \multicolumn{5}{|l|}{ Situação conjugal } \\
\hline Com companheiro & $17(81,0)$ & $67(74,5)$ & 1,00 & \\
\hline Sem companheiro & $04(19,0)$ & $23(25,6)$ & 1,45 & $0,53^{\ddagger}$ \\
\hline \multicolumn{5}{|l|}{ Tipo de parto } \\
\hline Normal & $12(57,0)$ & $40(44,0)$ & 1,00 & \\
\hline Cesárea & $09(43,0)$ & $50(56,0)$ & 1,66 & $0,29^{\ddagger}$ \\
\hline \multicolumn{5}{|l|}{ Primiparidade } \\
\hline Sim & $10(47,6)$ & $45(50,0)$ & 0,90 & $0,84^{\ddagger}$ \\
\hline Não & $11(52,4)$ & $45(50,0)$ & 1,00 & \\
\hline \multicolumn{5}{|l|}{ Histórico de Nascidos com Baixo Peso } \\
\hline Sim & $04(19,0)$ & $07(7,8)$ & 2,78 & $0,25^{* *}$ \\
\hline Não & $17(81,0)$ & $83(92,2)$ & 1,00 & \\
\hline \multicolumn{5}{|l|}{ Histórico de Nascidos Prematuros } \\
\hline Sim & $01(4,8)$ & $06(6,7)$ & 0,70 & $1,00 * *$ \\
\hline Não & $20(95,2)$ & $84(93,3)$ & 1,00 & \\
\hline \multicolumn{5}{|l|}{ Uso de tabaco durante a gestação } \\
\hline Sim & $01(4,8)$ & $02(2,2)$ & 2,2 & $0,94^{* *}$ \\
\hline Não & $20(95,2)$ & $88(97,8)$ & 1,00 & \\
\hline \multicolumn{5}{|l|}{ Uso de álcool durante a gestação } \\
\hline Sim & $11(52,4)$ & $11(12,2)$ & 7,9 & $0,00^{\ddagger}$ \\
\hline Não & $10(47,6)$ & $79(87,8)$ & 1,00 & \\
\hline TOTAL & $21(100,0)$ & $90(100,0)$ & & \\
\hline
\end{tabular}

* Nível de significância estatística: $\mathrm{p} \leq 0,05 .{ }^{* *}$ Teste Exato de Fischer. ${ }^{\ddagger}$ Teste $\chi^{2}$ de Pearson. 
Tabela 2. Características relacionadas à atenção pré-natal dos grupos caso e controle $(n=111)$.

\begin{tabular}{|c|c|c|c|c|}
\hline \multirow{2}{*}{ Características } & Casos $(n=21)$ & Controles $(n=90)$ & \multirow{2}{*}{ OR } & \multirow{2}{*}{$\mathbf{p}^{*}$} \\
\hline & n (\%) & n (\%) & & \\
\hline \multicolumn{5}{|l|}{ № de Consultas no Pré-Natal } \\
\hline$<6$ & $13(61,9)$ & $32(35,5)$ & 2,94 & $0,02^{\ddagger}$ \\
\hline$\geq 6$ & $08(38,1)$ & $58(65,5)$ & 1,00 & \\
\hline \multicolumn{5}{|l|}{ Infecção urinária } \\
\hline Sim & $09(43,0)$ & $39(39,3)$ & 0,98 & $0,96^{\ddagger}$ \\
\hline Não & $12(57,0)$ & $51(56,7)$ & 1,00 & \\
\hline \multicolumn{5}{|l|}{ Hipertensão } \\
\hline Sim & $04(19,0)$ & $09(10,0)$ & 2,11 & $0,42^{* *}$ \\
\hline Não & $17(81,0)$ & $81(90,0)$ & 1,00 & \\
\hline \multicolumn{5}{|l|}{ Outras doenças } \\
\hline Sim & $10(47,6)$ & $22(24,4)$ & 0,76 & $0,06^{\ddagger}$ \\
\hline Não & $11(32,4)$ & $68(75,6)$ & 1,00 & $0,06^{\ddagger}$ \\
\hline \multicolumn{5}{|l|}{ IMC pré-gestacional } \\
\hline$<18,5 \mathrm{~kg} / \mathrm{m}^{2}$ & $02(9,5)$ & $10(11,1)$ & 0,64 & 0,58 \\
\hline$\geq 18,5$ e $<25 \mathrm{~kg} / \mathrm{m}^{2}$ & $12(57,0)$ & $26(28,9)$ & 1,00 & \\
\hline$\geq 25 \mathrm{e}<30 \mathrm{~kg} / \mathrm{m}^{2}$ & $03(14,3)$ & $26(28,9)$ & 0,28 & 0,05 \\
\hline$\geq 30 \mathrm{~kg} / \mathrm{m}^{2}$ & $03(14,3)$ & $06(6,7)$ & 1,85 & 0,41 \\
\hline \multicolumn{5}{|l|}{ Tomou sulfato ferroso } \\
\hline Sim & $20(95,2)$ & $81(90,0)$ & 1,00 & \\
\hline Não & $01(4,8)$ & $09(10,0)$ & 2,22 & $0,79 * *$ \\
\hline \multicolumn{5}{|l|}{ Tomou ácido fólico } \\
\hline Sim & $20(95,2)$ & $82(91,1)$ & 1,00 & \\
\hline Não & $01(4,8)$ & $08(8,9)$ & 1,95 & $0,92^{* *}$ \\
\hline \multicolumn{5}{|l|}{ Tomou medicamento abortivo } \\
\hline Sim & $04(19,0)$ & $04(4,4)$ & 5,05 & $0,04 * *$ \\
\hline Não & $17(81,0)$ & $86(95,6)$ & 1,00 & \\
\hline TOTAL & $21(100,0)$ & $90(100,0)$ & & \\
\hline
\end{tabular}

* Nível de significância estatística: $p \leq 0,05$. ** Teste Exato de Fischer. ‡ Teste $\chi 2$ de Pearson.

Tabela 3. Características relacionadas à higiene bucal materna durante a gestação dos grupos caso e controle $(n=111)$.

\begin{tabular}{|c|c|c|c|c|}
\hline \multirow{2}{*}{ Características } & Casos $(n=21)$ & Controles $(n=90)$ & \multirow{2}{*}{ OR } & \multirow{2}{*}{$\mathbf{p}^{*}$} \\
\hline & n (\%) & n (\%) & & \\
\hline \multicolumn{5}{|l|}{ Já perdeu dentes } \\
\hline Sim & $11(52,4)$ & $53(58,9)$ & 0,76 & \multirow[t]{2}{*}{$0,58^{\ddagger}$} \\
\hline Não & $10(47,6)$ & $37(41,1)$ & 1,00 & \\
\hline \multicolumn{5}{|l|}{ Teve problemas para mastigar alimentos } \\
\hline Sim & $10(47,6)$ & $12(13,3)$ & 0,57 & \multirow[t]{2}{*}{$0,24^{\ddagger}$} \\
\hline Não & $11(52,4)$ & $78(86,7)$ & 1,00 & \\
\hline \multicolumn{5}{|c|}{ Sente-se satisfeita com aparência de sua boca } \\
\hline Sim & $07(33,3)$ & $16(17,7)$ & 1,00 & \multirow[b]{2}{*}{$0,20^{* *}$} \\
\hline Não & $14(66,7)$ & $74(82,3)$ & 2,31 & \\
\hline \multicolumn{5}{|c|}{ Seus dentes ou gengivas ficaram sensíveis a alimentos e líquidos } \\
\hline Sim & $14(66,7)$ & $24(26,7)$ & 5,50 & \multirow[t]{2}{*}{$0,00^{\ddagger}$} \\
\hline Não & $07(33,3)$ & $66(73,3)$ & 1,00 & \\
\hline \multicolumn{5}{|l|}{ Uso do fio dental } \\
\hline $\operatorname{Sim}$ & $06(28,6)$ & $35(38,9)$ & 1,00 & \multirow[b]{2}{*}{$0,37^{\ddagger}$} \\
\hline Não & $15(71,4)$ & $55(61,1)$ & 0,62 & \\
\hline \multicolumn{5}{|l|}{ Visita ao dentista } \\
\hline Sim & $20(95,2)$ & $72(80,0)$ & 1,00 & \multirow{3}{*}{$0,16^{\ddagger}$} \\
\hline Não & $01(4,8)$ & $18(20,0)$ & 5,00 & \\
\hline TOTAL & $21(100,0)$ & $90(100,0)$ & & \\
\hline
\end{tabular}

* Nível de significância estatística: $p \leq 0,05$. ** Teste Exato de Fischer. $\neq$ Teste $\chi 2$ de Pearson. 
Na Tabela 2 são apresentadas as características relacionadas à atenção pré-natal entre os grupos de comparação. Observou-se que a chance de indivíduos nascerem com BPN é 2,94 vezes maior para as mães que fizeram menos de 6 consultas no pré-natal e de 5,05 vezes maior para aquelas que usaram medicamento abortivo na gestação, dados estes com significância estatística. Por sua vez, o IMC das mães entre 25 e $30 \mathrm{~kg} / \mathrm{m}^{2}$, revelou-se como fator protetor à ocorrência de BPN/prematuridade (OR: 0,28; $p=0,05$ ).

Quando observadas as covariáveis relacionadas ao comportamento em saúde bucal (Tabela 3), verificou-se que os grupos do estudo foram semelhantes para todas as características investigadas, com exceção da sensibilidade dos dentes ou gengiva a alimentos e líquidos durante a gestação, vez que foi estimada uma chance de 5,50 vezes maior desta condição, para as mães que tiveram filhos com $\mathrm{BPN}$ quando comparadas àquelas do grupo controle (OR: $5,50 ; p<0,001)$.

\section{DISCUSSÃO}

Os resultados demonstraram que as puérperas expostas ao pré-natal inadequado apresentaram quase três vezes mais chance de terem RN com peso menor que $2500 \mathrm{~g}$ e parto prematuro, e que a frequência desses eventos ocorreram em mulheres com idade menor ou igual a 25 anos. No entanto, quando comparados os grupos, a ocorrência do evento foi mais acentuada naquelas com idade maior que 25 anos. A literatura tem demonstrado que em caso de idade mais elevada, é esperada uma maior exposição a doenças crônicas como hipertensão arterial sistêmica, pré-eclâmpsia, anemia, rotura prematura de membranas, além de diversos outros fatores de risco potenciais citados na literatura ${ }^{13-18}$. Acrescenta-se ainda que a associação entre fatores sociodemográficos e peso ao nascer é bem estabelecida, o que pode ser devido à íntima relação das causas sociais com a desnutrição e infecções. A associação entre fatores sociodemográficos e peso ao nascer é bem estabelecida, o que pode ser devido à íntima relação das causas sociais com a desnutrição e infecções ${ }^{19}$.

Já em relação a gestação em adolescentes, observou-se em um hospital público de uma cidade do Paquistão, onde foi realizado um estudo pela World Health Organization ${ }^{14}$ uma associação entre recém nascidos com baixo peso (RNBP) e idade materna abaixo de 20 anos (9,9\%), num contexto de pior condição socioeconômica ( $30 \%$ de analfabetismo materno e $40 \%$ de prevalência de anemia) mesmo após ajuste para varáveis consideradas potencialmente confundidoras. Apesar das adolescentes apresentarem maiores prevalências de prénatal inadequado, além de má nutrição e menor renda familiar deve-se considerar o efeito da boa condição dos indicadores sociodemográficos da região, nesses resultados ${ }^{15-17}$.
No presente estudo, a baixa escolaridade não se mostrou associada a uma maior chance de BPN, confirmando os achados de Haiddar et al. ${ }^{18}$ e Silva ${ }^{20}$. Por outro lado, a frequência da ocupação materna durante a gestação no grupo controle $(28,9 \%)$ foi maior quando comparado aos casos (19\%). Porém, não foram detectadas diferenças relevantes, provavelmente dado o baixo poder do estudo. Em relação a hábitos de vida deletérios, foi observada uma prevalência de etilistas da ordem de 19,8\%. Embora já seja estabelecido que o etilismo materno durante a gestação possa acarretar perda expressiva do crescimento fetal ${ }^{9}, 12,2 \%$ das puérperas investigadas consumiram álcool durante a gravidez, sendo que $52,4 \%$ destas fazia parte do grupo caso.

Quanto à influência do acompanhamento de pré-natal na prevenção de desfechos gestacionais adversos, a maioria das pesquisas disponíveis na literatura especializada tem empregado indicadores quantitativos para estudo de tal relação ${ }^{21}$. No entanto, autores têm ressaltado a possibilidade de que vieses de seleção e o modo de organização de serviços de saúde devam ser considerados visto que os mesmos podem conduzir a efeito de confundimento. Isso implica na necessidade de uma estratégia robusta para seleção de participantes e sua devida alocação seja no grupo de casos ou no de controles, bem como a inclusão de variáveis que permitam medir a interferência da estrutura organizacional dos serviços de assistência ao pré-natal22-23. No presente estudo foi significativa a associação com número de consultas pré-natais $(p=0,02)$. Verificou-se que menos de seis consultas aumentou a chance para a ocorrência de BPN, o que converge com os estudos de Nobile et al..$^{24} \mathrm{e}$ Vohr et al. ${ }^{25}$. Contudo, Almeida et al. ${ }^{13}$ relatam que sete consultas ainda são insuficientes para prevenir RNBP.

Um outro fator de risco citado na literatura refere-se à história de RNBP em gestação prévia ${ }^{16}$. Por sua vez, o parto cesariano não mostrou significativamente relacionado com o BPN, mas vale destacar que este foi mais frequente que o parto natural no grupo controle que no grupo caso (56\% vs $43 \%$ ). No entanto, os estudos prévios encontraram tal associação. Segundo Almeida ${ }^{19}$, o parto cesariano aumentou a ocorrência de BPN entre os anos de 1982 e 2004 em Pelotas (RS) durante o qual a proporção de RNBP mais que dobrou. Da mesma forma, na cidade de Campinas (SP), a cesariana foi contribuinte importante para ocorrência de BPN ${ }^{10}$.

A literatura tem indicado também que infecções crônicas da gestante tem efeito negativo no peso ao nascer. Nesse estudo, a frequência de sensibilidade gengival nos casos $(66,7 \%)$ foi maior que nos controles $(26,7 \%)$, embora as diferenças não tenham apresentado significância estatística. Esse achado clínico provavelmente sugere a presença de inflamação em tecidos periodontais que frequentemente 
se apresenta mais elevada na gravidez dada a influências hormonais gestacionais ${ }^{26,27}$. Bassani et al. ${ }^{28}$ encontraram uma menor prevalência da afeção periodontal/gengival, em puérperas com filhos de peso normal quando comparadas às mães de RNBP.

Diante de resultados ainda insuficientes disponíveis na literatura acerca da temática, é importante refletir sobre as possíveis explicações para as taxas de BPN/prematuridade. Um ponto importante refere-se à possibilidade de vieses de confusão. Para minimizar a ocorrência de tal erro, investigações em geral têm incluindo entre as variáveis estudadas, aquelas consideradas potencialmente confundidoras. Nesse sentido, a presente pesquisa mediu as diferenças entre os grupos de comparação relativas a componentes sociodemográficos, de estilo de vida, de condições de saúde geral e bucal, bem como de acesso a serviços de saúde.

Não obstante as questões supracitadas, os achados desta pesquisa devem ser avaliados com cautela. Primeiramente, devem ser observadas as limitações epidemiológicas do delineamento metodológico escolhido. É sabido que as investigações de caso-controle estão sujeitas a vieses de seleção dos grupos, bem como de vieses de memória devido ao seu caráter retrospectivo, além do possível constrangimento da puérpera durante a entrevista. Ademais, ressalta-se que o presente estudo pode não ter tido poder suficiente para detectar as reais diferenças entre os grupos, dado que o tamanho da amostra empregada foi pequeno, uma vez que se trata de estudo preliminar. O estudo maior se embasará nos resultados desse estudo preliminar de forma a refinar o método, reduzir vieses e agregar maior robustez aos seus resultados.

No entanto, a despeito das limitações dessa investigação, e desse ainda ser um tema controverso na literatura, os resultados apresentados podem contribuir para o corpo de evidências em questão, ao revelar que aquelas mulheres que tinham o hábito de consumir bebida alcoólica, que realizaram menos de seis consultas pré-natais e utilizaram medicação abortiva, tiveram maior chance do bebê nascer com baixo peso e prematuro. Assim, os entraves metodológicos não suprimem a relevância desta pesquisa, mas sinalizam a necessidade de mais estudos que melhor esclareçam os fatores de riscos associados a estes eventos, através de arranjos metodológicos robustos e com maior poder de análise.

\section{REFERÊNCIAS}

1. Organização Mundial de Saúde. Meeting of Advisory Group on Maternal Nutrition and Low Birthweight [Internet]. Genebra: OMS; 2002 [acesso em 2016]. Disponível em: http://www.who.int/ nutrition/topics/lbw_strategy_background.pdf
2. Ebersole JL, Novak MJ, Michalowicz BS, Hodges JS, Steffen MJ, Ferguson JE, Diangelis A, Buchanan W, Mitchell DA, Papapanou $\mathrm{PN}$. Sistemic immune responses in pregnancy and periodontitis: relationship to pregnancy outcomes in the obstetrics and periodontal therapy (OPT) study. J Periodontol. 2009;80(6):953-60. http:// dx.doi.org/10.1902/jop.2009.080464

3. Cruz SS, Costa M da CN, Gomes-Filho IS, Barreto ML. Periodontal therapy for pregnant women and cases of low birthweight: An intervention study. Pediatr Int. 2010;52(1):57-64. http://dx.doi. org/10.1111/j.1442-200X.2009.02888.x

4. Ministério da Saúde (BR). Secretaria de Atenção à Saúde. Departamento de Ações Programáticas Estratégicas. Manual de vigilância do óbito infantil e fetal e do comitê de prevenção do óbito infantil e fetal. Brasília: Ministério da Saúde; 2009.

5. Victora CG, Aquino EML, Leal MC, Monteiro CA, Barros FC, Szwarcwald CL. Maternal and child health in Brazil: progress and challenges. Lancet. 2011; 377(9780):1863-76. http://dx.doi. org/10.1016/S0140-6736(11)60138-4

6. Malta DC, Duarte EC, Escalante JJC, Almeida MF, Sardinha LMV, Macário EM. Mortes evitáveis em menores de um ano, Brasil, 1997 a 2006: contribuições para a avaliação de desempenho do Sistema Único de Saúde. Cad Saúde Pública. 2010;26(3):481-91. http:// dx.doi.org/10.1590/S0102-311X2010000300006

7. United Nations Children's Fund and World Health Organization, Low Birthweight: Country, regional and global estimates [Internet]. New York: UNICEF; 2004 [acesso em 2016]. Disponível em: http:// www.unicef.org/publications/index_24840.html

8. Ministério da Saúde (BR). Secretaria de Atenção à Saúde. Departamento de Ações Programáticas Estratégicas. Atenção à Saúde do Recém-Nascido: Guia para os profissionais de saúde. 2ª ed. Brasilia-DF: Ministério da Saúde; 2012.

9. Melo ASO, Assunção PL, Gondim SSR, Carvalho DF, Amorim MMR, Benicio MHD, Cardoso MAA. Estado nutricional materno, ganho de peso gestacional e peso ao nascer. Rev Bras Epidemiol. 2007;10(2):249-57. http://dx.doi.org/10.1590/S1415$790 \times 2007000200012$

10. Carniel EF, Zanolli ML, Antônio MARGM, Morcillo AM. Determinantes do baixo peso ao nascer a partir das Declarações de Nascidos Vivos. Rev Bras Epidemiol. 2008;11(1):169-79. http:// dx.doi.org/10.1590/S1415-790X2008000100016

11. Geib LTC, Fréu CM, Brandão M, Nunes ML. Determinantes sociais e biológicos da mortalidade infantil em coorte de base populacional em Passo Fundo, Rio Grande do Sul. Ciênc Saúde Coletiva. 2010;15(2):363-70. http://dx.doi.org/10.1590/S141381232010000200011

12. Beck S1, Wojdyla D, Say L, Betran AP, Merialdi M, Requejo JH, Rubens C, Menon R, Van Look PF. The worldwide incidence of preterm birth: a systematic review of maternal mortality and morbidity. Bull World Health Organ. 2010;88(1):31-8. http://dx.doi. org/10.2471/BLT.08.062554

13. Barros FC, Victora CG, Matijasevich A, Santos IS, Horta BL, Silveira MF, Barros AJD. Preterm births, low birth weight, and intrauterine growth restriction in three birth cohorts in Southern Brazil: 1982, 1993 and 2004. Cad Saúde Pública. 2008;24 Suppl 3:S390-8. http://dx.doi.org/10.1590/S0102-311X2008001500004 
14. World Health Organization. Major causes of death in neonates and children under five GLOBAL 2008 (revised). The World Health Organization Statistic [Internet]. 2011 [acesso em 2016]. Disponível em: http://www.who.int/gho/publications/world_health_statistics/ EN_WHSO8_Full.pdf

15. Kramer MS. Determinants of low birth weight: methodological assessment and meta-analysis. Bull World Health Organ. 1987;65(5):663-737.

16. Valero De Bernabé J, Soriano T, Albaladejo R, Juarranz M, Calle ME, Martínez D, Domínguez-Rojas V. Risk factors for low birth weight: a review. Eur J Obstet Gynecol Reprod Biol. 2004;116(1):3-15. http:// dx.doi.org/10.1016/j.ejogrb.2004.03.007

17. Araújo BF, Tanaka ACA. Fatores de risco associados ao nascimento de recém-nascidos de muito baixo peso em uma população de baixa renda. Cad Saúde Pública. 2007;23(12):2869-77. http:// dx.doi.org/10.1590/S0102-311X2007001200008

18. Haiddar FH, Oliveira UF, Nascimento LFC. Escolaridade materna: correlação com os indicadores obstétricos. Cad Saúde Pública. 2001;17(4):1025-9. http://dx.doi.org/10.1590/S0102$311 \times 2001000400037$

19. Almeida MF, Novaes HMD, Alencar GP, Rodrigues LC. Mortalidade neonatal no município de São Paulo: influências do peso ao nascer e de fatores sócio-demográficos e assistências. Rev Bras Epidemiol. 2002;5(1):93-106. http://dx.doi.org/10.1590/S1415790X2002000100011

20. Silva AAM, Bettiol H, Barbieri MA. Which factors could explain the low birth weight paradox? Rev Saúde Pública. 2006;40 (4):648-55. http://dx.doi.org/10.1590/S0034-891020060005 00014
21. Badshah S, Mason L, McKelvie K, Payne R, Lisboa PJ. Risk factors for low birthweight in the public-hospitals at Peshawar, NWFP-Pakistan. BMC Public Health. 2008;8:197. http://dx.doi.org/10.1186/14712458-8-197

22. Sclowitz IKT, Santos IS. Fatores de risco na recorrência do baixo peso ao nascer, restrição de crescimento intra-uterino e nascimento prétermo em sucessivas gestações: um estudo de revisão. Cad Saúde Pública. 2006 Jun;22(6):1129-36. http://dx.doi.org/10.1590/S0102$311 \times 2006000600002$

23. Silveira DS, Santos IS. Adequação do pré-natal e peso ao nascer: uma revisão sistemática. Cad Saúde Pública. 2004;20(5):1160-8. http://dx.doi.org/10.1590/S0102-311X2004000500009

24. Nobile CG, Raffaele G, Altomare C, Pavia M. Influence of maternal and social factors as predictors of low birth weight in Italy. BMC Public Health. 2007;7:192. http://dx.doi.org/10.1186/1471-2458-7-192

25. Vohr BR, Tyson JE, Wright LL, Perritt RL, Li L, Poole WK; NICHD Neonatal Research Network. Maternal age, multiple birth, and extremely low birth weight infants. J Pediatr. 2009;154(4):498503.e2. http://dx.doi.org/10.1016/j.jpeds.2008.10.044

26. Bertolini PFR, Biondi Filho O, Niero BG, Saraceni CHC, Splendore SMG, Pomílio A, Guanais MAB. Medicina periodontal e a mulher: a importância do seu conhecimento para uma abordagem preventiva por ginecologistas/obstetras e cirurgiões-dentistas. Rev Ciênc Med PUCCAMP 2007;16:175-85.

27. Soory M. Hormonal factors in periodontal disease. Dent Update. 2000;27(8):380-3.

28. Bassani DG, Olinto MT, Kreiger N. Periodontal disease and perinatal outcomes: a case-control study. J Clin Periodontol. 2007;34(1):31-9. http://dx.doi.org/10.1111/j.1600-051X.2006.01012.x 